\title{
Backing-up Fuzzy Control of a Truck-trailer Equipped with a Kingpin Sliding Mechanism
}

\author{
G. Siamantas and S. Manesis \\ Electrical \& Computer Engineering Dept., University of Patras, Patras, Greece \\ gsiama@upatras.gr;stam.manesis@ece.upatras.gr
}

\begin{abstract}
For articulated vehicles met in robotics and transportation fields, even for an experienced operator, backing-up leads usually to jack-knifing. This paper presents a fuzzy logic controller for back-driving a truck-trailer vehicle into a predefined parking task. The truck-trailer link system is equipped with a kingpin sliding mechanism acting as an anti-jackknife excitation input. By applying fuzzy logic control techniques, precise system model is not required. The developed controller with thirty four rules works well as the presented simulation results demonstrate the avoidance of jack-knife and the accuracy of the backing-up technique.
\end{abstract}

\section{Introduction}

Control of the backward movement of a truck and trailer vehicle, called docking task, is known to be a typical nonlinear control problem. The difficulty of the control system design not only causes the dynamics to be nonlinear, but also emphasizes the inherent physical limitations of the system such as the jackknife phenomenon, a mathematical model of which is discussed in (Fossum and Lewis 1981). The control system under investigation is not only nonlinear but also nonholonomic. Backward movement control of computer simulated truck-trailers using various types of intelligent control e.g. fuzzy control, neural control and neurofuzzy or genetic algorithm-based control (Yang et al. 2006, Kiyuma et al. 2004, Riid and Rustern 2001), has been reported. The backing-up control of a trucktrailer is considered in (Park et al. 2007) as a system with time delay. A fuzzy knowledge-based control for backing multi-trailer systems is also considered in (Riid et al. 2007). The kingpin sliding mechanism, used in current work for backward stabilization, has been also used for off-tracking elimination in multiarticulated vehicles (Manesis et al. 2003). In this paper the kingpin sliding mechanism is used mainly as an active anti-jackknife steering mechanism for backward motion. The mathematical model of the considered system is different and more

Please use the following format when citing this chapter:

Siamantas, G. and Manesis, S., 2009, in IFIP International Federation for Information Processing, Volume 296; Artificial Intelligence Applications and Innovations III; Eds. Iliadis, L., Vlahavas, I., Bramer, M.; (Boston: Springer), pp. 373-378. 
complex from that of a truck-trailer without a kingpin sliding mechanism. The precise mathematical description offers only an initial guidance for writing the fuzzy rules of the controller.

\section{System description - Fuzzy Logic Controller Design}

The system under consideration is a truck-trailer vehicle with on-axle sliding-able connection. Its geometry along with the directions of all defined angles and axes are shown in Fig. 1. The major difference with a typical truck-trailer system is the use of a sliding kingpin on the rear axle of the tractor.

The nomenclature of the considered model is: $\left(x_{0}, y_{0}\right)$ coordinates of the truck's rear axle midpoint, $\left(x_{1}, y_{1}\right)$ coordinates of the trailer's rear axle midpoint, $\theta_{0}$ angle of the longitudinal axis of the truck w.r.t. the horizontal axis, $\theta_{1}$ angle of the longitudinal axis of the trailer w.r.t. the horizontal axis, $S$ length of the kingpin slide, $l_{0} \quad$ truck's wheelbase length, $l_{1}$ length between the trailer's rear axle midpoint and the hinge joint, $\varphi$ truck's steering wheels turning angle, $U_{1}$ truck velocity, $S, U_{2}$ rate of change of the kingpin sliding, $\alpha$ angle between the horizontal axis and the straight line that passes from the axes origin and the $\left(x_{1}, y_{1}\right)$ point, $d$ distance of the $\left(x_{1}, y_{1}\right)$ point from the horizontal axis (same as $\left.y_{1}\right), \alpha-\theta_{1}$ angle between the trailer's longitudinal axis and the line that passes from the axes origin and the $\left(x_{1}, y_{1}\right)$ point, $\theta_{0}-\theta_{1}$ angle between the longitudinal axes of the truck and the trailer. The kinematic equations of the system are the following:

$$
\begin{aligned}
x_{0} & =U_{1} \cos \theta_{0} \\
y_{0} & =U_{1} \sin \theta_{0} \\
\theta_{0} & =\frac{U_{1}}{l_{0}} \tan \varphi \\
\theta_{1} & =\frac{1}{l_{1}}\left(U_{1}+S \frac{U_{1}}{l_{0}} \tan \varphi\right) \sin \left(\theta_{0}-\theta_{1}\right)-\sin ^{-1}\left(\frac{S}{l_{1}}\right) \\
S & =U_{2} \\
x_{1} & =x_{0}-l_{1} \cos \theta_{1}+S \sin \theta_{0} \\
y_{1} & =y_{0}-l_{1} \sin \theta_{1}-S \cos \theta_{0}
\end{aligned}
$$

The $-\sin ^{-1}\left(S / l_{1}\right)$ term was added to take into account the change of angle $\theta_{1}$ in case where the velocity $U_{1}$ is zero and the rate of change of the kingpin sliding 
$U_{2}$ is not zero. The above system model will be used in the evaluation of the designed fuzzy logic controller performance through simulations.

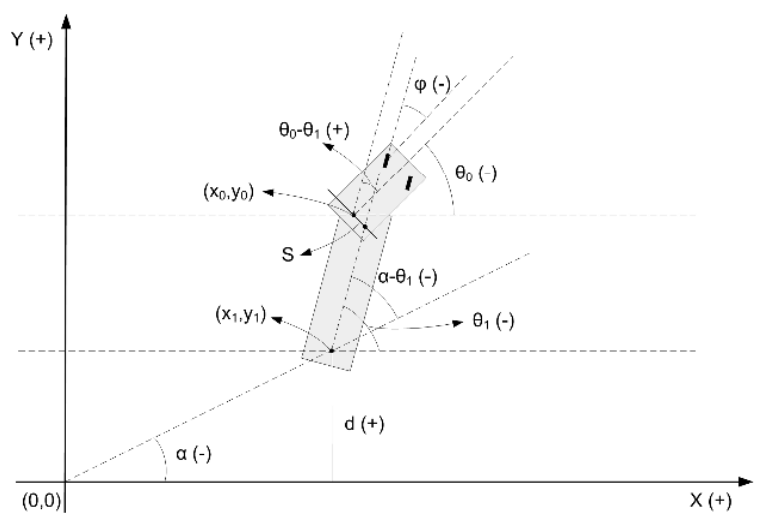

Fig. 1. System geometry

The main objective of this Fuzzy Logic Controller (FLC) is the backward motion control of the truck and trailer system to follow a target line, a procedure similar to parking. In the design of the fuzzy logic controller we used 4 inputs $\left(\alpha-\theta_{1}, \theta_{0}-\theta_{1}, d, S\right)$ and 2 outputs $(\varphi, S)$. From the four inputs the first three are needed for achieving the control objective and the fourth one $(S)$ is needed to make $S$ zero when the first two inputs become zero. The input and output variables were defined to have 5 membership functions each: NL: Negative Large, NS: Negative Small, ZE: Zero, PS: Positive Small, PL: Positive Large. The normalized degree of membership function diagrams for each input or output variable are shown in Fig. 2. For the input variables the NS, ZE, PS membership functions are triangular and the NL, PL are trapezoidal. The output variables have all 5 membership functions triangular.

The fuzzy logic controller has been developed by defining 34 logical rules shown in Fig. 3. These rules are consistent with the following evaluation strategies: (1) the truck's steering wheels must turn in such a way as to make the system move towards the direction that makes the angle $\alpha-\theta_{1}$ zero, (2) if the angles $\alpha-\theta_{1}$ and $\theta_{0}-\theta_{1}$ belong to the ZE degree, i.e. the truck-trailer system is moving towards the axes origin and the angle between the truck and the trailer is close to zero, then we can turn the truck's steering wheels in such a way as to minimize the distance $d$ from the horizontal axis, (3) if the angle $\theta_{0}-\theta_{1}$ is large (NL, PL degrees) and to avoid jackknifing, regardless of the values of all other variables, the steering wheels should be turned in such a way as to minimize the angle $\theta_{0}-\theta_{1}$, (4) the kingpin sliding should have a direction opposite to the centrifugal direction and must be proportional to the angle $\theta_{0}-\theta_{1}$. This action will provide greater cor- 
rection margin to the steering wheels to avoid jackknifing, (5) if the angles $\alpha-\theta_{1}$ and $\theta_{0}-\theta_{1}$ belong to the $\mathrm{ZE}$ degree, i.e. the truck-trailer system is moving towards the axes origin and the angle between the truck and the trailer is close to zero, then we can make the kingpin sliding distance zero by applying opposite $S$ proportionally to $S$. The linguistic rules that were used have the general form:

\section{IF $\left[\mathrm{INP}_{1}\right.$ is $\left.\mathrm{MF}_{\mathrm{INP} 1}\right]$ AND $\left[\mathrm{INP}_{2}\right.$ is $\mathrm{MF}_{\mathrm{INP} 2}$ ] THEN [OUT 1 is $\left.\mathrm{MF}_{\mathrm{OUT} 1}\right]$}

The fuzzy operator AND and the implication method were defined as minimum. The centroid defuzzification method was used. The output aggregation method was defined as sum. We have chosen the sum method instead of the maximum method because it fits better to the cumulative control method imposed from strategy no. 2 used in controlling the distance $d$ over the control of the $\alpha-\theta_{1}$ angle.
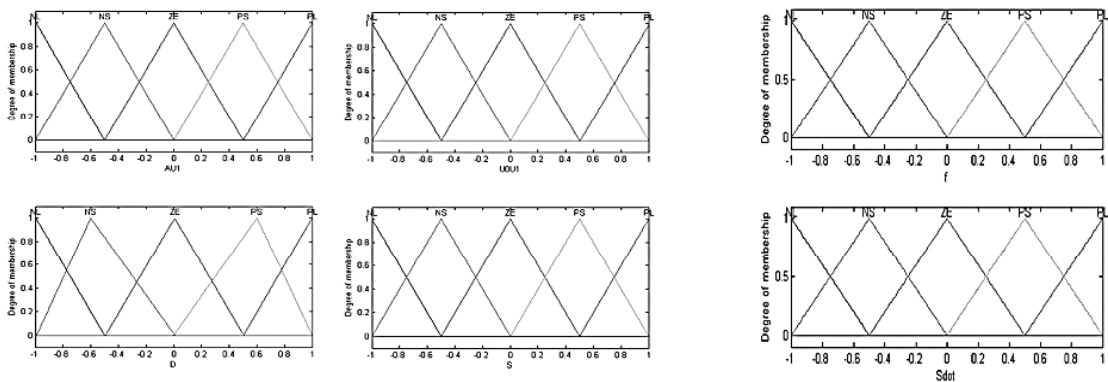

Fig. 2. Normalized degree of membership function diagrams of input-output variables

\begin{tabular}{|c|c|c|c|c|c|c|}
\hline \multicolumn{2}{|c|}{$\varphi / \dot{S}$} & \multicolumn{5}{c|}{$\theta_{0}-\theta_{1}$} \\
\hline$\alpha-\theta_{1}$ & $d$ & NL & NS & ZE & PS & PL \\
\hline NL & & $N L / P L$ & $P S / P S$ & $P S / Z E$ & $P L / N S$ & $P L / N L$ \\
\hline NS & & $N L / P L$ & $Z E / P S$ & $P S / Z E$ & $P S / N S$ & $P L / N L$ \\
\hline & NL & & & $P L / Z E$ & & \\
\cline { 2 - 7 } ZE & & & $P S / Z E$ & & \\
\cline { 2 - 7 } & & $N L / P L$ & $N S / P S$ & $Z E / Z E$ & $P S / N S$ & $P L / N L$ \\
\cline { 2 - 7 } & PS & & & $N S / Z E$ & & \\
\cline { 2 - 7 } & PL & & & $N L / Z E$ & & \\
\hline PS & & $N L / P L$ & $N S / P S$ & $N S / Z E$ & $Z E / N S$ & $P L / N L$ \\
\hline PL & & $N L / P L$ & $N L / P S$ & $N S / Z E$ & $N S / N S$ & $P L / N L$ \\
\hline
\end{tabular}

\begin{tabular}{|c|c|c|}
\hline \multicolumn{2}{|c|}{$\dot{S}$} & $\theta_{0}-\theta_{1}$ \\
\hline$\alpha-\theta_{1}$ & $S$ & ZE \\
\hline & NL & $P L$ \\
\hline & NS & $P S$ \\
\hline $\mathbf{Z E}$ & ZE & $Z E$ \\
\hline & PS & $N S$ \\
\hline & PL & $N L$ \\
\hline
\end{tabular}

Fig. 3. Fuzzy Logic Controller logical rules 


\section{Simulations, Motion Restrictions, Advantages of Kingpin Sliding}

To verify the effectiveness of the proposed fuzzy controller we performed several tests in a simulation environment. The simulation model was based on equations (1) with the following assumptions: $U_{1}$ is $-1 \mathrm{~m} / \mathrm{sec}, l_{0}$ is $4 \mathrm{~m}, l_{1}$ is $6 \mathrm{~m}, \varphi$ varies between $-50^{\circ}$ and $50^{\circ}, \varphi$ varies between $-30 \%$ sec and $30^{\circ} / \mathrm{sec}, S$ varies between $0.6 \mathrm{~m}$ and $0.6 \mathrm{~m}$ and $S$ varies between $-0.1 \mathrm{~m} / \mathrm{sec}$ and $0.1 \mathrm{~m} / \mathrm{sec}$. Because the designed membership function diagrams are normalized we used gains in the inputs and outputs to change their universes of discourse scales.

Several simulation tests were performed, from different system initial positions and directions, with and without kingpin sliding control. In Fig. 4, we see the system response from initial conditions $\left(x_{0}, y_{0}, \theta_{0}, \theta_{1}\right)=(60,-10,45,-45)$. We see that kingpin sliding control does not have big influence in system response as far as concerning the trajectory of the system. The difference is that we have greater angle margin for the control of a possible jackknife situation in the case where kingpin sliding control is used. This can be shown from simulations and also from the system geometry where we see that with a kingpin sliding along the centripetal direction we gain approximately $\sin ^{-1}\left(S / l_{1}\right)=5.74^{\circ}$ in $\theta_{0}-\theta_{1}$ angle and by extension to the steering wheel angle $\varphi$. The positive effects of this angle gain are demonstrated in Fig. 5 where the initial conditions are $(60,20,45,-45)$. In these responses the turn limit of the truck's steering wheels is between $-35^{\circ}$ and $35^{\circ}$. It is obvious that without kingpin sliding control the system enters a jackknife condition, Fig. 5 (left). The system with kingpin sliding control avoids jackknife, Fig. 5 (right).

It should be noted that when the truck-trailer system is moving backwards the advantages of kingpin sliding control appear during sliding towards the centripetal direction in contrast with the system moving forward where the advantages appear during sliding towards the centrifugal direction.
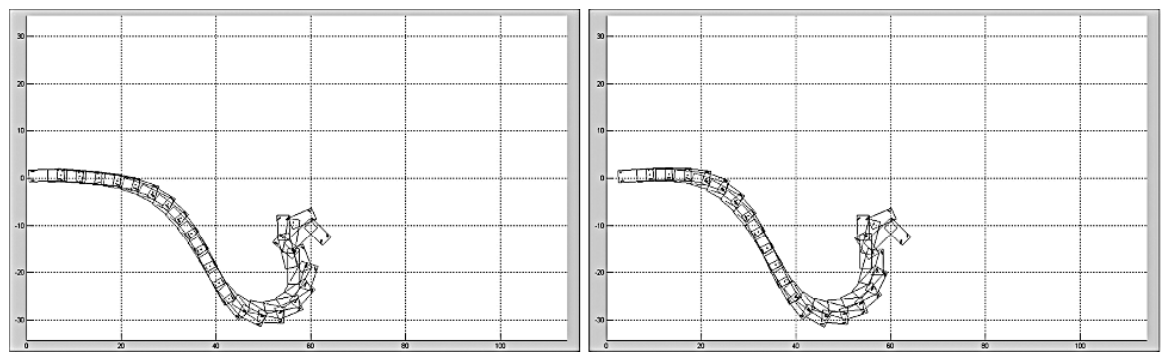

Fig. 4. System response from initial conditions $(60,-10,45,-45)$. Without sliding control (left), with sliding control (right) 

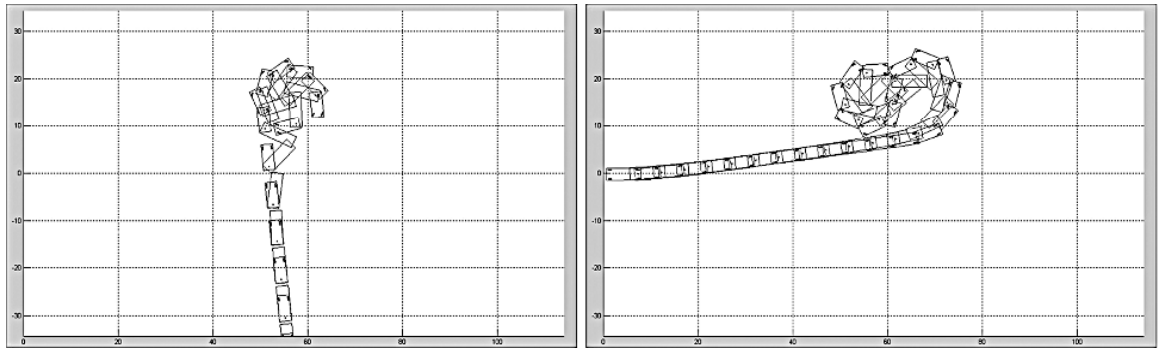

Fig. 5. System response from initial conditions $(60,20,45,-45)$ and $\left(-35^{\circ}, 35^{\circ}\right)$ steering wheel angle limit. Without sliding control (left - jackknife), with sliding control (right)

\section{Conclusions}

A fuzzy logic controller was designed for the backward motion of a truck-trailer system with on-axle kingpin sliding. Various tests were conducted to test the controller effectiveness. The controller showed good performance with the system starting from various initial conditions. The use of kingpin sliding towards the centripetal direction has no adverse effect in system response while it reduces the maximum turn of the truck's steering wheels when the system is maneuvering backwards. This gives greater steering wheel turn margin for the truck to avoid jackknife.

\section{References}

1. Fossum TV., Lewis GN. A mathematical model for trailer-truck jackknife. SIAM Review (1981); vol.23, no.1: 95-99.

2. Kiyuma A., Kinjo H., Nakazono K., Yamamoto T. Backward control of multitrailer systems using neurocontrollers evolved by a genetic algorithm. Proceedings 8th International Symposium on Artificial Life and Robotics (2004); pp.9-13.

3. Manesis S., Koussoulas N., Davrazos G., On the suppression of off-tracking in multiarticulated vehicles through a movable junction technique. Journal of Intelligent and Robotic Systems (2003); vol.37: pp.399-414.

4. Park C.W., Kim B.S., Lee J., Digital stabilization of fuzzy systems with time-delay and its application to backing up control of a truck-trailer. International Journal of Fuzzy Systems, (2007), vol.9, pp.14-21.

5. Riid A., Ketola J., Rustern E., Fuzzy knowledge-based control for backing multi-trailer systems. Proceedings IEEE Intelligent Vehicles Symposium, (2007), pp.498-504.

6. Riid A., Rustern E. Fuzzy logic in control: Track backer-upper problem revisited. Proceedings 10th IEEE International Conference on Fuzzy Systems (2001); vol.1: pp.513-516.

7. Yang X., Yuan J., Yu F. Backing up a truck and trailer using variable universe based fuzzy controller. Proceedings IEEE International Conference on Mechatronics and Automation (2006); pp. 734-739. 